\title{
Color superconductivity in high density QCD
}

\author{
Roberto Casalbuoni \\ Dipartimento di Fisica dell' Universita' di Firenze and Sezione INFN, Via G. Sansone 1, 50019 \\ Sesto Fiorentino (Firenze), Italy.E-mail: casalbuoni@fi.infn.it
}

It is now a well established fact that at zero temperature and sufficiently high densities quark matter is a color superconductor $[1,2]$. The study starting from first principles was done in $[3,4,5]$. At densities much higher that the masses of the quarks $u, d$ and $s$, the favored state is the so-called Color-Flavor-Locking (CFL) state, whereas at lower densities the strange quark decouples and the relevant phase is called two-flavor color superconducting (2SC).

An interesting possibility is that in the interior of compact stellar objects (CSO) some color superconducting phase may exist. In fact we recall that the central densities for these stars could be up to $1 \div 1.5 \mathrm{fm}^{-3}$, whereas the temperature is of the order of tens of $\mathrm{keV}$. However the usual assumptions leading to show, for instance, that with three flavors the favored state is CFL, should now be reviewed. Matter inside a CSO should be electrically neutral and should not carry any color. Also conditions for $\beta$ equilibrium should be fulfilled. As far as color is concerned, it is possible to impose a simpler condition, that is color neutrality, since in [6] it has been shown that there is no free energy cost in projecting color singlet states out of color neutral states. With the further observation that using $\beta$-equilibrium one has $\mu_{e}=-\mu_{Q}$, where $\mu_{e}$ and $\mu_{Q}$ are the chemical potentials associated to the electrons and to the electric charge respectively, we see that all the previous conditions can be satisfied by requiring that the electric-charge, $T_{3}$ and $T_{8}$ densities vanish. This is equivalent to require that the derivatives of the free energy with respect to the corresponding chemical potentials are zero:

$$
\frac{\partial \Omega}{\partial \mu_{e}}=\frac{\partial \Omega}{\partial \mu_{3}}=\frac{\partial \Omega}{\partial \mu_{8}}=0
$$

A color superconducting state is also characterized by a non-zero expectation value of a diquark operator which depends, in the homogeneous case, on several constants, the gaps. The free energy is evaluated by starting from a microscopic description of the quark interaction, which is usually assumed to be a four-fermi interaction. Of course this is an approximation to the real case, but it turns out to be quite effective. In this way one gets the free-energy as a function of the chemical potentials and of the energy gaps, $\Delta_{i}$. Therefore, besides requiring the conditions (1), we have to minimize the free energy also with respect to $\Delta_{i}$

$$
\frac{\partial \Omega}{\partial \Delta_{i}}=0
$$


Another important point to be considered in these applications is the mass of the strange quark, since the relevant chemical potentials are of order $400-500 \mathrm{MeV}$. Both the strange quark mass and the $\beta$-equilibrium in conjunction with electrical neutrality have the effect of producing a mismatch in the Fermi momenta of the pairing fermions. For increasing mismatch the BCS pairing mechanism is lost and the system can undergo a phase transition either to the normal state or to a different phase.

Let us review how the mismatch in the Fermi momenta is originated. Consider a massive fermion and a massless one at the same chemical potential $\mu$. The corresponding Fermi momenta are

$$
p_{F_{1}}=\sqrt{\mu^{2}-M^{2}} \approx \mu-\frac{M^{2}}{2 \mu}, \quad p_{F_{2}}=\mu
$$

Therefore the mismatch is of order $M^{2} / \mu$. For the second instance, consider that $\beta$ equilibrium requires the chemical potential for the electrons to be the opposite of the chemical potential associated to the electric charge. Then, the neutrality condition determines uniquely $\mu_{e}$ in terms of the other chemical potentials. Since $\mu_{e}$ is not arbitrary one is forced to consider the free energy along the neutrality line rather than long a line of fixed $\mu_{e}$. The result of this analysis, at first sight, is a little bit surprising, since where one has unstable phases moving along lines of constant $\mu_{e}$, now one gets stable phases along neutrality lines [7].

If we characterize the mismatch in terms of the difference among effective chemical potentials, call it $2 \delta \mu$, the typical spectrum of quasi-fermions is $E= \pm \delta \mu+$ $\sqrt{(p-\mu)^{2}+\Delta^{2}}$. We see that if $\delta \mu \geq \Delta$ gapless modes are present in correspondence of momenta $p=\mu \pm \sqrt{\delta \mu^{2}-\Delta^{2}}$. The point $\delta \mu=\Delta$ plays a special role, since the energy cost for pairing the fermions belonging to Fermi spheres with a mismatch is $2 \delta \mu$ whereas the gain for pairing is $2 \Delta$. Therefore when $\delta \mu>\Delta$ fermions start to lose their BCS pairing.

Examples of the previous situation are the gapless phases g2SC [7] and gCFL [8,9]. In the g2SC phase the fermion condensate has the same structure as in the 2SC phase, but there is a mismatch $\delta \mu=\mu_{e} / 2$, and correspondingly a phase transition from 2SC to $\mathrm{g} 2 \mathrm{SC}$ at $\mu_{e}=2 \Delta$. The analysis shows that the g2SC phase is stable along the neutrality line. In this particular example the quarks involved are the quark up and the quark down, so there is no effect from the strange quark mass, but only from the interplay of $\beta$ equilibrium and electrical neutrality.

The gCFL has a fermionic condensate given by

$$
\left\langle 0\left|\psi_{a L}^{\alpha} \psi_{b L}^{\beta}\right| 0\right\rangle=\Delta_{1} \varepsilon^{\alpha \beta 1} \varepsilon_{a b 1}+\Delta_{2} \varepsilon^{\alpha \beta 2} \varepsilon_{a b 2}+\Delta_{3} \varepsilon^{\alpha \beta 3} \varepsilon_{a b 3}
$$

whereas in CFL, $\Delta_{1}=\Delta_{2}=\Delta_{3}$. In this case the mass strange quark mechanism for the mismatch plays the driving role. In fact, the mismatch among the blue-down and greenstrange quarks is given by $M_{s}^{2} /(2 \mu)$ and a phase transition between the CFL and the gCFL (where $\Delta_{3}>\Delta_{2}>\Delta_{1}$ ) occurs exactly at $M_{s} / \mu=2 \Delta$. From the analysis made in [9] it turns out that gCFL is the favored phase over $2 \mathrm{SC}$ and g2SC up to about $M_{s}^{2} / \mu \approx 130$ $\mathrm{MeV}$, for a choice of the gap $\Delta$ in CFL given by $25 \mathrm{MeV}$.

In presence of a mismatch among the Fermi momenta of the pairing fermions there is another interesting possibility which has been considered in the literature, that is the so 
called LOFF phase [10] (see also the reviews [11]). In this case the mechanism proposed is a different way of pairing in which each of the pairing fermions stays close to its own Fermi surface. As a consequence the pair has a non zero total momentum leading to a breaking of translational and rotational invariance [12]. This phase is particularly interesting since it may give rise to a crystalline structure. This possibility was widely explored in [13] within the context of a Ginzburg-Landau expansion. Since the validity of this expansion in the present context is not completely justified the result of the analysis was a conjecture about the most favored structure, a face centered cube. In [14] a different approximation to the problem was proposed. This approximation holds in a region far from a second order transition, and in this sense is complementary to the Ginzburg Landau expansion. It was found that when the BCS phase is lost a LOFF phase with a crystalline structure corresponding to an octahedron (or a body centered

cube) takes place. This happens at $\delta \mu=\Delta / \sqrt{2}$. This phase remains the favored one up to $\delta \mu \approx 0.95 \Delta$ where the face-centerd cube crystal becomes energetically favored. Then this phase persists up to $1.32 \Delta$, when the system goes back to the normal phase. According to the authors of ref. [9] this would imply (extrapolating the results of ref. [14] obtained in the $2 \mathrm{SC}$ case), that the LOFF phase should take place at about $M_{s}^{2} / \mu \approx 120$ $\mathrm{MeV}$ up to $225 \mathrm{MeV}$. If this would be the case the phases 2SC and g2SC would play no role since increasing $M_{s}$ one would go from CFL to gCFL through a second order transition and then via a first order one to the LOFF phase.

\section{REFERENCES}

1. B. Barrois, Nuclear Physics B129, 390 (1977); S. Frautschi, Proceedings of workshop on hadronic matter at extreme density, Erice 1978; D. Bailin and A. Love, Physics Report 107, 325 (1984).

2. M. Alford, K. Rajagopal, and F. Wilczek, Phys. Lett. B 422, 247 (1998); R. Rapp, T. Schafer, E. V. Shuryak and M. Velkovsky, Phys. Rev. Lett. 81, 53 (1998).

3. D.T. Son, Phys. Rev. D 59, 094019 (1999); T. Schäfer and F. Wilczek, Phys. Rev. D 60, 114033 (1999); D.K. Hong, V.A. Miransky, I.A. Shovkovy, and L.C.R. Wijewardhana, Phys. Rev. D 61, 056001 (2000); S.D.H. Hsu and M. Schwetz, Nucl. Phys. B572, 211 (2000); W.E. Brown, J.T. Liu, and H.-C. Ren, Phys. Rev. D 61, 114012 (2000).

4. R.D. Pisarski and D.H. Rischke, Phys. Rev. D 61, 051501 (2000).

5. I.A. Shovkovy and L.C.R. Wijewardhana, Phys. Lett. B 470, 189 (1999); T. Schäfer, Nucl. Phys. B575, 269 (2000).

6. P. Amore, M. C. Birse, J. A. McGovern and N. R. Walet, Phys. Rev. D 65, 074005 (2002) [arXiv:hep$\mathrm{ph} / 0110267]$.

7. I. Shovkovy and M. Huang, Phys. Lett. B564, 205 (2003) [arXiv:hep-ph/0302142].

8. M. Alford, C. Kouvaris and K. Rajagopal, Phys. Rev. Lett. 92, 222001 (2004) [arXiv:hep$\mathrm{ph} / 0311286]$.

9. M. Alford, C. Kouvaris and K. Rajagopal, [arXiv:hep-ph/0406137].

10. A. I. Larkin and Yu. N. Ovchinnikov, Sov. Phys. JETP 20 (1965), 762; P. Fulde and R. A. Ferrell, Phys. Rev. 135 (1964), A550.

11. R. Casalbuoni and G. Nardulli, Rev. of Mod. Phys. 76, 263 (2004) [arXiv:hep-ph/0305069]; J. A. Bowers, [arXiv:hep-ph/0305301].

12. M. G. Alford, J. A. Bowers and K. Rajagopal, Phys. Rev. D63 (2001) 074016, [arXiv:hep$\mathrm{ph} / 0008208]$.

13. J. A. Bowers and K. Rajagopal, Phys. Rev. D66 (2002) 065002, [arXiv:hep-ph/0204079].

14. R. Casalbuoni, M. Ciminale, M. Mannarelli, G. Nardulli, M. Ruggieri and R. Gatto, [arXiv:hep$\mathrm{ph} / 0404090]$. 